\title{
The holographic vortex lattice using the circular cell method
}

\author{
Gianni Tallarita ${ }^{a}$ and Roberto Auzzi ${ }^{b, c}$ \\ ${ }^{a}$ Departamento de Ciencias, Facultad de Artes Liberales, Universidad Adolfo Ibáñez, \\ Diagonal Las Torres, Santiago 7941169, Chile \\ ${ }^{b}$ Dipartimento di Matematica e Fisica, Università Cattolica del Sacro Cuore, \\ via Musei 41, 25121 Brescia, Italy \\ ${ }^{c}$ INFN, Sezione di Perugia, \\ via A. Pascoli, 06123 Perugia, Italy \\ E-mail: gianni.tallarita@uai.cl, rauzzi@gmail.com
}

ABSTRACT: We investigate vortex lattice solutions in a holographic superconductor model in asymptotically $\mathrm{AdS}_{4}$ spacetime which includes the gravitational backreaction of the vortex. The circular cell approximation, which is known to give a good result for several physical quantities in the Ginzburg-Landau model, is used. The critical magnetic fields and the magnetization curve are computed. The vortex lattice profiles are compared to expectations from the Abrikosov solution in the regime nearby the upper critical magnetic field $H_{2 c}$ for which superconductivity is lost.

KEYworDs: Holography and condensed matter physics (AdS/CMT), Solitons Monopoles and Instantons, AdS-CFT Correspondence

ARXIV EPRINT: 1909.05932 


\section{Contents}

1 Introduction 1

2 Theoretical setting 3

2.1 The normal state 5

3 The vortex lattice $\quad 6$

3.1 Metric ansatz 6

$\begin{array}{lll}3.2 & \text { Boundary conditions and solutions } & 7\end{array}$

3.3 The critical magnetic field limit $H_{2 c}$

4 Free energy and magnetization $\quad 10$

5 Discussion 12

\section{Introduction}

An important and generic property of higher temperature superconductors is the presence of a strange metal state found just above the superconducting critical temperature. The transport properties of strange metals are very different from the ones of conventional Fermi liquid. In particular, the standard quasi-particle picture does not give a useful description of the physics of the system [1,2]. An interesting class of models without a quasi-particles description can be built using the AdS/CFT correspondence. The correspondence maps a strongly interacting quantum system in the boundary to a classical gravity problem in the bulk, and so it provides a controlled environment in which to study strongly coupled systems.

Since Abrikosov's seminal work [3], the magnetic properties of type II superconductors have been the subject of many experimental and theoretical studies (see [4] for a review). In this phase magnetic flux penetrates the superconductor by forming vortices, which are arranged in lattice geometries. Using several microscopic techniques, these periodic arrays of vortices have been experimentally studied in the lab both for conventional and for higher temperature superconductors.

The Ginzburg-Landau (GL) theory is a very useful macroscopic description of superconductors (see [5] for a textbook) which can be used to model the Abrikosov vortex lattice in a quantitative way. Strictly speaking, the GL theory is valid only close to the critical temperature; indeed, it can be derived from the Bardeen-Cooper-Schrieffer (BCS) theory just in this regime. Given the experimental importance of vortex lattices in higher-temperature superconductors, it is important to theoretically study vortex lattices also in theoretical situations where no quasi-particle picture is available. Holographic superconductors [6-9] 
provide a controlled theoretical laboratory to explore situations where the quasi-particle approximation is not applicable, and so they may give precious hints on the behaviour of vortex lattices in non-conventional superconductors.

Vortices in holographic superconductors have been studied by many authors. Most of the early studies [10-16] neglect the gravitational backreaction of the vortex solution. This is a well justified approximation in the regime where the scalar condensate is small. A systematic study of gravitational backreaction in the case of a single vortex was performed in [17]. This analysis allowed to systematically compute thermodynamic properties of the vortex.

The study of vortex lattices is more complicated because there is no cylindrical symmetry, and so one needs to solve a partial differential equation with an extra dynamical variable. Without backreaction, a study of the holographic vortex lattice was initiated in [18]. Vortex lattices with backreaction were studied in the $\mathrm{AdS}_{2} \times R^{2}$ geometry in [19, 20]: this geometry describes the near horizon limit of extremal magnetic Reissner-Nordstrom black holes, and so it is relevant for the zero temperature limit of a holographic vortex lattice. The vortex lattice in a holographic model with SU(2) gauge field was studied in [21]. Vortex lattices in holographic superfluid were studied in [22, 23].

The problem of constructing the fully backreacted holographic vortex lattice, valid for all ranges of magnetic fields and temperatures, remains therefore an unsolved problem. This is not surprising, the problem involves hard numerical computations, with complicated starting ansatze for the metric and matter fields. In this paper we will provide an approximate solution to this problem using the circular cell method (CCM) [24], which is a technique already used for vortex lattices in the Landau-Ginzburg framework [25-27].

The CCM approximates the full geometrical lattice solution by replacing each cell of the lattice with a circular one of the same area (see figure 1). This dramatically simplifies the problem as one can use a cylindrical symmetry ansatz to simplify the calculation. For the case of standard Abrikosov lattices, the method is extremely accurate over the whole range of magnetic fields, with several physical quantities such as critical magnetic field and magnetization curve differing by percent level between the full geometrical result and the CCM [25-27]. This remarkable result serves as motivation to use this method in the holographic context. We will however quantify the validity of the approximation, at least in the previously mentioned limit of critical fields, where an analytic solution is available.

The paper is organized as follows: in section 2 we provide an introduction to the theoretical setting with which we will work throughout the paper. In section 3 we will apply the CCM to the holographic vortex lattice and we will compute the magnetization curve of the superconductor. In section 3.3 we will compare the circular cell approximation to the Abrikosov solution in the limit of critical magnetic field $H_{2 c}$. We conclude in section 5 . 


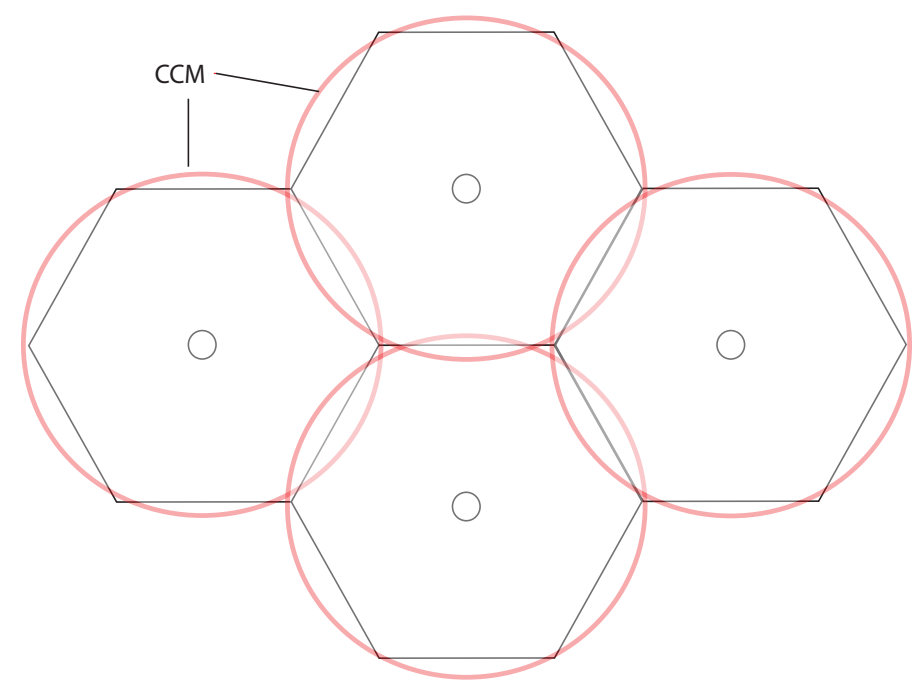

Figure 1. Schematic representation of Circular Cell Method.

\section{Theoretical setting}

We will consider the same model of holographic superconductor in asymptotically $\mathrm{AdS}_{4}$ spacetime as in [17]. The bulk lagrangian is:

$$
S_{A N O}=\frac{1}{16 \pi G_{N}} \int d^{4} x \sqrt{-g}\left[R+\frac{6}{L^{2}}-\frac{1}{2} F_{\mu \nu} F^{\mu \nu}-2\left(D_{\mu} \phi\right)\left(D^{\mu} \phi\right)^{\dagger}-2 V\left(|\phi|^{2}\right)\right],
$$

where $L$ is the AdS radius and

$$
\begin{array}{rlrl}
V\left(|\phi|^{2}\right) & =-\frac{2}{L^{2}}|\phi|^{2}\left(1-\frac{1}{2}|\phi|^{2}\right) . \\
F_{\mu \nu} & =\partial_{\mu} A_{\nu}-\partial_{\nu} A_{\mu}, & D_{\mu} \phi=\partial_{\mu} \phi-i q A_{\mu} \phi .
\end{array}
$$

Here $D_{\mu}$ denotes the combination of the gravity and $\mathrm{U}(1)$ gauge covariant derivatives. We use slightly unconventional field normalizations: in our units $\phi$ and $A_{\mu}$ are both dimensionless, while $q$ has the same dimension as energy ( $q L$ is instead dimensionless). It turns out that the dimensionless quantity $q L$ indirectly characterizes the ratio between the magnetic penetration and the coherence length: indeed, as shown in [17], for $q L=1$ the vortex is in the type II regime while for $q L=2$ it is type I.

The extrema of the potential (2.2) are

$$
\begin{array}{lll}
|\phi|=0, & V=0, & m_{\phi}^{2}=-\frac{2}{L^{2}}, \\
|\phi|=1, & V=-\frac{1}{L^{2}}, & m_{\phi}^{2}=\frac{4}{L^{2}} .
\end{array}
$$

We will consider the first of these AdS vacua, whose metric is:

$$
d s^{2}=\frac{L^{2}}{z^{2}}\left(-d t^{2}+d z^{2}+d r^{2}+r^{2} d \theta^{2}\right) .
$$


Nearby the boundary, the field $\phi$ has the following expansion

$$
\phi=\alpha z^{\Delta_{1}}+\beta z^{\Delta_{2}}+\ldots, \quad \Delta_{1}=1, \quad \Delta_{2}=2 .
$$

The dimensions $\Delta_{i}$ are the solutions of $m_{\phi}^{2} L^{2}=\Delta(\Delta-3)$.

In order to allow for a vortex solution, we first need to realize a phase with a non-zero homogeneous scalar field outside the vortex core. In the original holographic superconductor model $[7,8]$ a chemical potential was introduced as a boundary condition of the $A_{0}$ field, in order for the scalar field $\phi$ to condense in the bulk. It turns out that, in order to achieve the condensation of $\phi$ in a holographic model, one does not need to consider the complication of non-zero background charge density. As explained in [28], the condensation of $\phi$ can be achieved also in a neutral black hole background, using a Robin boundary condition of the following form:

$$
\beta=\kappa \alpha, \quad \kappa<0,
$$

which is dual to introducing a relevant double-trace deformation in the field theory side of the holographic duality $[29,30]$ :

$$
\Delta \mathcal{V}=\kappa \mathcal{O}^{\dagger} \mathcal{O}
$$

When $\kappa$ is negative, this term triggers the condensation of the $\mathcal{O}$ operator. We choose to perform our calculations in the double trace deformation because it avoids the necessity to deal with an extra field profile for $A_{0}(z)$, which reduces the number of equations that we have to solve.

With zero magnetic field, the critical temperature for condensation [28] is:

$$
T_{c}=\frac{3}{4 \pi} \frac{\Gamma(1 / 3)^{3}}{\Gamma(-1 / 3) \Gamma(2 / 3)^{2}} \kappa \approx-0.62 \kappa .
$$

The equations of motion resulting from the action in eq. (2.1) are:

$$
G_{\mu \nu}=0, \quad D_{\mu} F^{\mu \nu}=i q\left[\left(D^{\nu} \phi\right) \phi^{\dagger}-\left(D^{\nu} \phi\right)^{\dagger} \phi\right]=J_{\mu}, \quad g^{\mu \nu} D_{\mu} D_{\nu} \phi-V^{\prime}\left(|\phi|^{2}\right) \phi=0,
$$

where

$$
G_{\mu \nu}=R_{\mu \nu}+\frac{3}{L^{2}} g_{\mu \nu}-\left[\left(D_{\mu} \phi\right)\left(D_{\nu} \phi\right)^{\dagger}+\left(D_{\nu} \phi\right)\left(D_{\mu} \phi\right)^{\dagger}+g_{\mu \nu} V\left(|\phi|^{2}\right)+F_{\mu}^{\sigma} F_{\sigma \nu}-\frac{g_{\mu \nu}}{4} F^{\rho \sigma} F_{\rho \sigma}\right] .
$$

For the dual conformal field theory interpretation, it is crucial to specify the boundary condition for the $\mathrm{U}(1)$ at $z=0[13,34]$. We denote by $\vec{B}$ the four dimensional gauge curvature tangent to the boundary, so that involves just $d x_{i} \wedge d x_{j}$ terms, where $x_{i}=(t, r, \theta)$. The $\vec{E}$ components instead involve terms such as $d x_{i} \wedge d z$, where $z$ is the $\mathrm{AdS}_{4}$ normal coordinate. If one chooses Dirichlet boundary condition $(\vec{B}=0)$, the field theory dual is a superfluid; instead with a Neumann boundary condition $(\vec{E}=0)$ a dynamical gauge field appears in the boundary, and the field theory dual is a superconductor. The two choices of boundary condition are related by bulk S duality [34]. The solution that we will discuss in the next section has cylindrical symmetry, with angular coordinate $\theta$. The only 
non-zero component of the gauge field is $A_{\theta}$, which has the following expansion nearby the boundary:

$$
A_{\theta}=a_{\theta}+z J_{\theta}+\mathcal{O}\left(z^{2}\right),
$$

where $z$ is the Fefferman-Graham (FG) coordinate for the asymptotically $\mathrm{AdS}_{4}$ backreacted metric. In order to impose the Neumann boundary condition, we will set $J_{\theta}=0$ in the boundary condition of the partial differential equation.

\subsection{The normal state}

In general, the vacuum state of our system with arbitrary temperature and applied magnetic field must be determined by solving the complicated non-linear system of PDE in eq. (2.10). In the regime above the upper applied critical magnetic $H_{2 c}$ for which superconductivity is lost, the $\phi$ condensate is zero and the magnetic field $\vec{B}$ is spatially uniform. The system then is described by the magnetic Reissner-Nordstrom (RN) black brane solution:

$$
d s^{2}=\frac{L^{2}}{z^{2}}\left(-f(z) d t^{2}+\frac{d z^{2}}{f(z)}+d \vec{x}^{2}\right)
$$

where

$$
f(z)=1-\left(1+\frac{z_{h}^{4} B^{2}}{2 L^{2}}\right)\left(\frac{z}{z_{h}}\right)^{3}+\frac{z_{h}^{4} B^{2}}{2 L^{2}}\left(\frac{z}{z_{h}}\right)^{4}, \quad A=B d x \wedge d y,
$$

and $z_{h}$ is the position of the horizon. The Hawking temperature $T$ is

$$
T=\frac{1}{4 \pi z_{h}}\left(3-\frac{z_{h}^{4} B^{2}}{2 L^{2}}\right) .
$$

In order to find $z_{h}$ as a function of $B, T$, one has to solve a quartic equation:

$$
z_{h}^{4}+z_{h}\left(\frac{8 \pi T L^{2}}{B^{2}}\right)-\frac{6 L^{2}}{B^{2}}=0 .
$$

The solution to eq. (2.16) can be written in compact form in two different limits:

$$
\begin{cases}\text { for } \sqrt{\frac{B}{L}} \ll T & z_{h}=\frac{3}{4 \pi T}, \\ \text { for } \sqrt{\frac{B}{L}} \gg T, & z_{h}=6^{1 / 4} \sqrt{\frac{L}{B}} .\end{cases}
$$

The $T \rightarrow 0$ limit corresponds to the extremal limit: in this case the near horizon metric is described by and $\mathrm{AdS}_{2} \times R^{2}$ metric.

The magnetization of the holographic dual system to the magnetic RN black brane in eq. (2.13) is [31-33]:

$$
M_{R S}=-\frac{\partial \hat{f}_{R S}}{\partial B}=-\frac{z_{h} B}{8 \pi G_{N}},
$$

where $\hat{f}_{R S}$ is the free energy density of the magnetic RS black brane. Evaluation of (2.18) gives:

$$
\begin{cases}\text { for } \sqrt{\frac{B}{L}} \ll T & M_{R S}=-\frac{3}{32 \pi^{2} G_{N}} \frac{B}{T}, \\ \text { for } \sqrt{\frac{B}{L}} \gg T, & M_{R S}=-\frac{6^{1 / 4}}{8 \pi G_{N}} \sqrt{L B} .\end{cases}
$$




\section{The vortex lattice}

In this section we dedicate our study to the range of applied magnetic fields in type II superconductors between the lower critical magnetic field $H_{c 1}$, where the magnetic field start to penetrate inside the superconductor, and the upper critical field $H_{c 2}$, where superconductivity is completely destroyed. In this regime the superconductor is pierced by a lattice of flux vortices.

The numerical solution of the backreacted holographic vortex lattice is in general a rather hard problem, because there is no cylindrical symmetry and one should solve a system of partial differential equations involving gravity in three dimensions.

In order to determine the critical magnetic fields and the magnetization curve in the GL model, it turns out that the CCM gives an excellent approximation [25-27]. In this section we apply the CCM to the an holographic vortex lattice with backreaction.

\subsection{Metric ansatz}

We denote by $R$ the radius of the circular cell in the boundary. The vortex cell area then is: $A_{\text {cell }}=\pi R^{2}$. In our units $R$ is dimensionless, since all scales in the model are set by $L$ and $\kappa$. We consider the following ansatz for the cylindrical symmetric metric in the bulk:

$$
\begin{aligned}
d s^{2}=\frac{L^{2}}{y^{2}}\{ & -Q_{1} y_{+}^{2}\left(1-y^{3}\right) d t^{2}+\frac{Q_{2}}{1-y^{3}} d y^{2} \\
& \left.+y_{+}^{2} Q_{4}\left(R d x+\frac{R x}{(1+R x)^{2}} y^{2} Q_{3} d y\right)^{2}+y_{+}^{2} Q_{5} R^{2} x^{2} d \theta^{2}\right\},
\end{aligned}
$$

where $Q_{1,2,3,4,5}$ are function of the AdS normal coordinate $y$ and of the vortex radial coordinate $x$. The boundary radial cylindrical coordinate is $r=R x$, in such a way that $x \in[0,1]$. This is very similar to the ansatz introduced in [17] for the single vortex case. The main difference is that the $\hat{x}$ coordinate used in [17] for the single vortex case is different from the $x$ coordinate used here. The relation is as follows:

$$
x=\frac{1}{R} \frac{\hat{x}}{1-\hat{x}}, \quad \hat{x} \in[0,1] .
$$

Here $y \in[0,1]$, with $y=0$ being the conformal boundary and $y=1$ the black hole horizon. Without loss of generality, we can set at the horizon $Q_{1}(x, 1)=Q_{2}(x, 1)$. With this choice of conventions, the Hawking temperature is:

$$
T=\frac{3 y_{+}}{4 \pi} .
$$

Note that the metric (3.1) is not in the Fefferman-Graham form, i.e.

$$
d s_{F G}^{2}=\frac{L^{2}}{z^{2}} d z^{2}+\gamma_{M N} d w^{M} d w^{M},
$$

where the capital latin letter denote the boundary coordinates $w^{M}=(t, \tilde{x}, \theta)$ and $y \approx y_{+} z$. The FG metric is useful to extract the expectation value of boundary quantities; the 
form (3.1) instead is more convenient to numerically solve the equations of motion (2.10). The change of variables which brings the metric (3.1) in FG form can be expanded as follows nearby the boundary

$$
y=y_{+} z+\sum_{i=2}^{\infty} a_{i}(\tilde{x}) z^{i}, \quad x=\tilde{x}+\sum_{i=1}^{\infty} b_{i}(\tilde{x}) z^{i},
$$

where some of the lowest order coefficients $\left(a_{i}, b_{i}\right)$ can be found in appendix $\mathrm{A}$ of [36].

The matter field profiles are taken as follows [17]:

$$
\phi=y e^{i n \theta}\left(\frac{R x}{1+R x}\right)^{n} Q_{6}(x, y), \quad A_{\theta}=L\left(\frac{R x}{1+R x}\right)^{2} Q_{7}(x, y) .
$$

For the purposes of this paper we are only interested in winding $n=1$ vortices, therefore we restrict to this value from here on. We will impose the boundary condition $\partial_{y} Q_{7}(x, 0)=0$ in order to describe a superconductor and not a superfluid, see eqs. (2.12) and (3.5).

The equations of motion (2.10) lead to a complicated set of coupled non-linear partial differential equations for the $Q_{i}(x, y)$ which must be solved subject to specific boundary conditions which we discuss below.

The equations cannot be solved by standard numerical procedures in their current form. To make the equations elliptic, the DeTurck method is adopted. This is explained in detail in [35] and we will not review it here. For our case the reference metric for the DeTurck procedure is chosen to be the same line element as in eq. (3.1) with

$$
Q_{1}=Q_{4}=Q_{5}=1, \quad Q_{3}=0, \quad Q_{2}=1-\tilde{\alpha} y(1-y)
$$

where the DeTurck parameter is fixed to $\tilde{\alpha}=16 \kappa / 9$, in order to avoid logarithmic tails in the near boundary Fefferman-Graham expansion of the metric. As a final note, it is crucial that the solutions found must satisfy the vanishing of the DeTurck vector $\xi^{a} \xi_{a}=0$, otherwise they are known as Ricci solitons. We have checked that this is the case for all the numerical solutions presented in this paper.

\subsection{Boundary conditions and solutions}

The main idea of the CCM is to use a cylindrical symmetry ansatz (see figure 1) to approximate an triangular or square Wigner-Seitz cell. In the GL model, the following conditions are imposed in order to reproduce the physical vortex lattice [24]:

- the flux of the magnetic field in each cell is the same as the flux of an elementary vortex

- the radial derivative of the scalar condensate and of the magnetic field vanishes at the boundary of the cell.

The coordinate $x=1$ corresponds to the boundary of our cell, therefore this is where we have to pay special attention to this boundary condition. The geometry of the cell array 
has to match together in a smooth way. This gives the following boundary conditions for the metric:

$$
\partial_{x} Q_{1}(1, y)=\partial_{x} Q_{2}(1, y)=\partial_{x} Q_{4}(1, y)=\partial_{x} Q_{5}(1, y)=0, \quad Q_{3}(1, y)=0 .
$$

The boundary conditions the gauge fields come from the physical requirement that the total magnetic flux inside a cell is $1 / q$, which from Stokes' theorem is equivalent to $A_{\theta}(x=$ 1) $=1 / q$, i.e.

$$
Q_{7}(1, y)=\frac{(1+R)^{2}}{q L R^{2}},
$$

Eq. (3.9) is also equivalent to the condition that the current

$$
J_{\mu}=i q\left(\phi^{\dagger} D_{\mu} \phi-\phi D_{\mu} \phi^{\dagger}\right)
$$

vanishes at the boundary of a cell. The circular cell method implies that we have to impose the condition $D_{x} \phi=0$, i.e.

$$
\partial_{x} Q_{6}(1, y)=-\frac{1}{1+R} Q_{6}(1, y)
$$

Note that the original boundary condition for the circular cell method involved setting the derivative of the magnetic field to zero at the boundary of the cell. The condition $\partial_{x}\left(F^{\mu \nu} F_{\mu \nu}\right)=0$ at the boundary of the cell follows from eqs. (3.8), (3.9).

The other boundary conditions are chosen as in [17]:

- $y=0$. At the conformal boundary we require the metric to tend to the black brane solution, therefore

$$
Q_{1}=Q_{2}=Q_{4}=Q_{5}=1, \quad Q_{3}=0 .
$$

The boundary condition on the scalar field is the Robin condition previously mentioned:

$$
\partial_{y} Q_{6}(x, 0)=\frac{\kappa_{1}}{y_{+}} Q_{6}(x, 0),
$$

where $\kappa_{1}$ is related to $\kappa$ and to the $\tilde{\alpha}$ parameter as follows:

$$
\kappa_{1}=\frac{\tilde{\alpha} y_{+}}{4} .
$$

The boundary condition on the gauge field is the one which corresponds to the holographic dual of a superconductor, i.e. $\partial_{y} Q_{7}(x, 0)=0$.

- $x=0$. These conditions in the vortex core are derived in the appendix of [17],

$$
\begin{aligned}
& \partial_{x} Q_{1}(0, y)=\partial_{x} Q_{2}(0, y)=\partial_{x} Q_{4}(0, y)=\partial_{x} Q_{5}(0, y)=0, \quad Q_{4}(0, y)=Q_{5}(0, y), \\
& \partial_{x} Q_{3}(0, y)=2 R Q_{3}(0, y), \quad \partial_{x} Q_{6}(0, y)=R Q_{6}(0, y) \quad \partial_{x} Q_{7}(0, y)=2 R Q_{7}(0, y) .
\end{aligned}
$$

- $y=1$. At the horizon, the only condition that one must satisfy is that $Q_{1}(x, 1)=$ $Q_{2}(x, 1)$. 


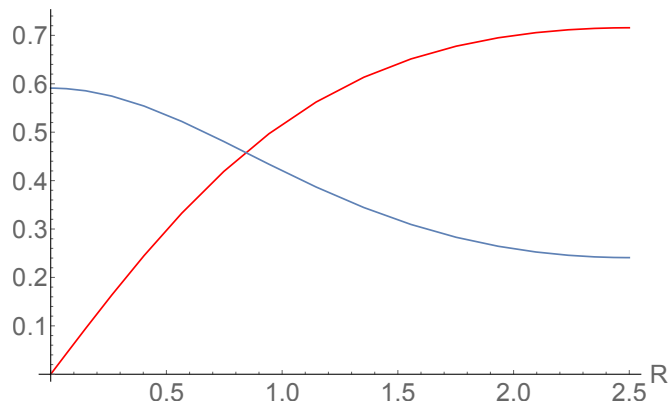

(a) $B$ (blue) and scalar field profiles.

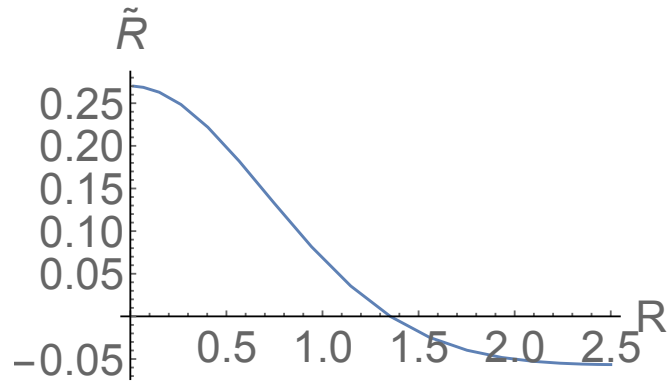

(b) Induced Ricci scalar at the horizon.

Figure 2. Some representative solutions of the superconductor case for $R=2.5$ and $q=1, \kappa=-1$, $y_{+}=1, G_{N}=1, L=1$. The solutions show the field profiles (scalar field in red and magnetic field in blue on the left, Ricci scalar on the right) in one cell of size $R$.

Some representative solutions are shown in figure 2, showing the profiles for the magnetic field, the scalar field and the induced horizon Ricci scalar in the vortex cell.

We have also checked that the induced Ricci scalar $\tilde{R}$ on the horizon is smooth $\left(\partial_{x} \tilde{R}=\right.$ 0 ) at $x=1$, and that there are therefore no gravitational singularities at the borders of the cell. Before proceeding to calculate the magnetization in the full magnetic field regime, we first make, in the next subsection, an important check on the accuracy of the circular cell approximation.

\subsection{The critical magnetic field limit $H_{2 c}$}

In the limit where the magnetic field approaches from below the upper critical field $H_{2 c}$, the scalar condensate is small and the authors of [18] derived an analytic solution for the holographic vortex lattice for a non-backreacting model. This solution was based on a different holographic model, namely one containing a chemical potential and no quartic potential. However, since the solution is separable in the bulk and spatial coordinates, we can use the spatial part of the solution as the spatial part of the solution to our model in this limit. This fact allows us to compare the full analytic result to our circular cell approximations taken close to the upper critical magnetic field limit.

The main idea is that nearby the critical magnetic field $H_{2 c}$ the AdS bulk equations are separable. The spatial part of the scalar condensate equation then reproduces the profiles found by Abrikosov [3].

This solution is of the form

$$
\phi\left(x_{1}, y_{2}, y\right)=\frac{\rho_{0}(y)}{L} \sum_{l=-\infty}^{l=\infty} c_{l} e^{i p_{l} x_{2}} \gamma_{0}\left(x_{1} ; p_{l}\right)
$$

where $\left(x_{1}, x_{2}\right)$ denote the two spatial directions and

$$
\gamma_{0}\left(x_{1} ; p_{l}\right)=\exp \left(-\frac{1}{2}\left(\frac{x_{1}}{r_{0}}-p r_{0}\right)^{2}\right), \quad p_{l}=\frac{2 \pi l}{a_{1} r_{0}}, \quad c_{l}=\exp \left(-i \frac{\pi a_{2}}{a_{1}^{2}} l^{2}\right),
$$




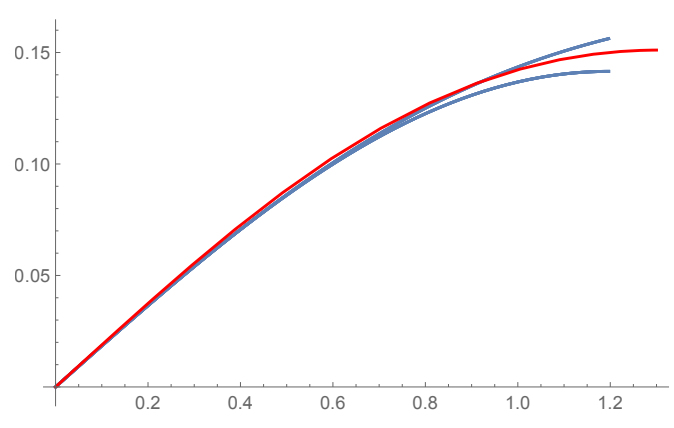

(a) Square.

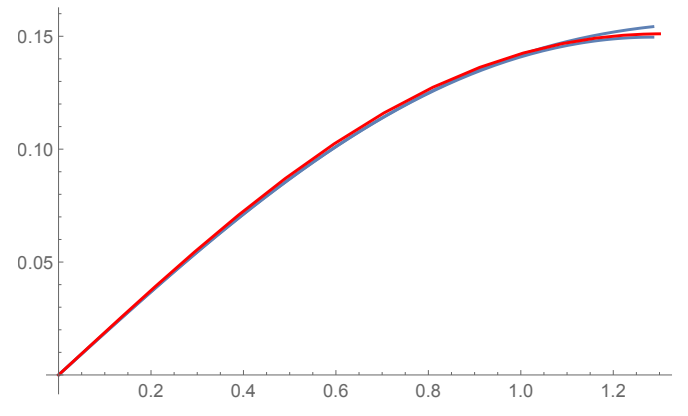

(b) Triangular.

Figure 3. Comparison of circular cell method with square and triangular analytic lattice for $H_{c 2}-B \approx 0.01$. The plot shows the scalar field profile $\phi$. Red line in between the other blue lines is the circular cell result. The other two correspond to the analytic solution in the $x_{1}$ (lower) and $x_{2}$ (higher) directions.

for constant $p$ and $r_{0} . \quad \rho_{0}(u)$ here denotes the profile of the solution on the AdS bulk direction. The parameters $a_{i}$ control the geometry of the lattice solution. For square lattices, we simply have $a_{1}=a_{2}$, while for triangular lattices the relation is

$$
\frac{a_{2}}{a_{1}}=\frac{a_{1}}{2}=3^{-1 / 4} \sqrt{\pi}
$$

In other words, for our model $\rho_{0}(y)$ will be different, but the rest of the solution is the same. The comparison of the spatial profiles at the boundary is independent of $\rho_{0}(y)$.

With this solution in hand, we performed an explicit check of the accuracy of our circular approximation by comparing vortex profiles in both $x_{1}$ and $x_{2}$ directions in this limit of our solution. There is a remarkable agreement between the two, see figure 3 , especially with the triangular case.

\section{$4 \quad$ Free energy and magnetization}

The free energy of the cell is defined by

$$
F=E-T S,
$$

where $E$ is the energy of our solution, $T$ is the temperature and $S$ is its entropy.

The energy can be computed by performing holographic renormalization in FG coordinates, see $[17,36]$ for details. The result for $E$ is:

$$
E=\frac{-y_{+}^{2}}{G} R^{2} \int_{0}^{1} x d x\left(\frac{3}{48} y_{+} Q_{1}^{(3)}(x)+\frac{17 y_{+} \alpha+160 \kappa_{1}}{256}\left(\frac{R x}{1+R x}\right)^{2} Q_{6}(x)^{2}\right)
$$

where $Q_{i}^{(k)}$ are the series expansion of the functions $Q_{i}$ in powers of $y$ around $y=0$ :

$$
Q_{i}=\sum_{k=0}^{\infty} Q_{i}^{(k)} y^{k}
$$


The entropy can be computed from the area of the horizon, see [17]:

$$
S=\frac{\pi}{2} y_{+}^{2} R^{2} \int_{0}^{1} x d x \sqrt{Q_{4}(x, 1) Q_{5}(x, 1)} .
$$

The final expression for the averaged free energy density is therefore

$$
\bar{f}=2 \pi F /\left(\pi R^{2}\right)=\frac{2(E-T S)}{R^{2}}
$$

where we included angular factors in the integration and divided by the cell area $\pi R^{2}$. We denote by $\bar{B}$ the averaged magnetic field in the cell:

$$
\bar{B}=\frac{1}{q} \frac{2}{R^{2}} .
$$

With these results in hand we can now proceed to calculate the magnetization for the holographic superconductor vortex array. This is the main advantage of using the circular cell method, as the calculation for the magnetization would otherwise involve solving the full three dimensional vortex array system, or resorting to magnetic field limits in which the system is tractable analytically. With this method, which as we saw is extremely accurate at least for the case of magnetic fields close to the upper critical value, we can extend the calculation to the whole range of magnetic field values.

Our superconducting boundary condition on $Q_{7}$ means the dual current $J_{\phi}=0$ and corresponds to infinite boundary gauge coupling $g \rightarrow \infty$. The finite value of the magnetization $M$, which is proportional to $g$ and $J_{\phi}$ then arises from a zero times infinity limit which is difficult to compute directly; it is more straightforward to use the definition of magnetization using free energy.

The applied magnetic field can be defined as the source to which the magnetic field is coupled and it can be extracted from the derivative of the free energy density with respect to $\bar{B}$ :

$$
H=\frac{\partial \bar{f}}{\partial \bar{B}}=-q \frac{R^{3}}{4} \frac{\partial \bar{f}}{\partial R} .
$$

This should be compared with a region of space where no superconductor is present, which is described by the Reissner-Nordstrom (RS) solution. We denote by $H_{R S}$ the applied magnetic field of the RS black brane with the same temperature and magnetic field $B=\bar{B}$ :

$$
H_{R S}=\frac{\partial \hat{f}_{R S}}{\partial B}=-M_{R S}
$$

see eq. (2.18). The magnetization can be expressed as the difference between the RS magnetic field and the applied magnetic field in the presence of the superconductor:

$$
M=H_{R S}-H .
$$

Numerical plots of this results, for varying values of $q$, are shown in figure 4 . These values are all chosen inside the type II parameter space. The type II/type I transition is close to $q L=2$. 


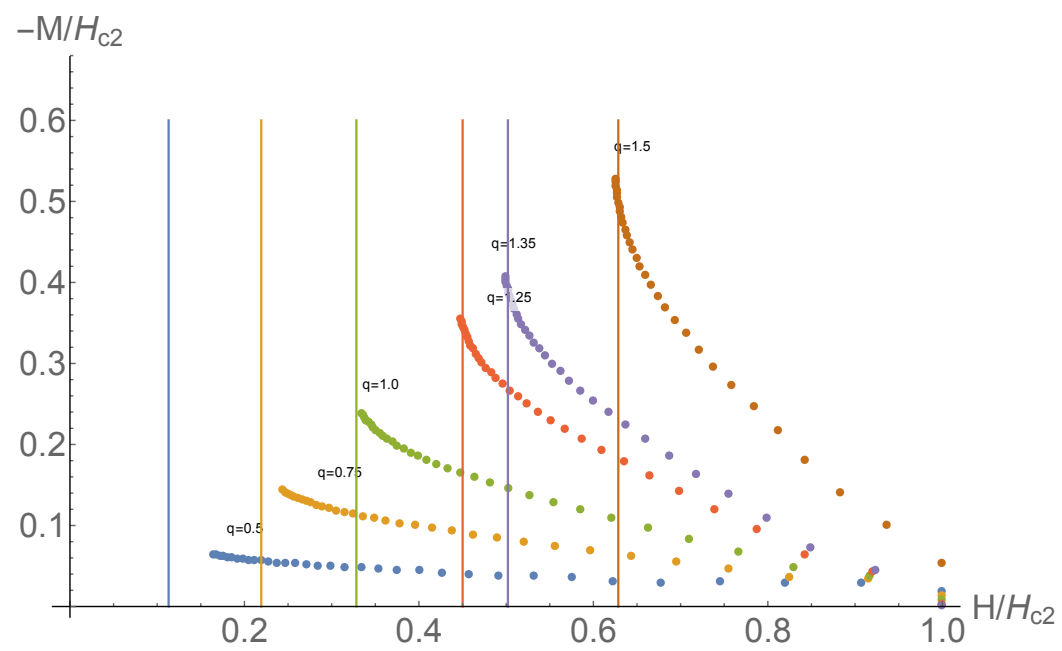

Figure 4. Magnetization plot for several values of $q$ including values of $H_{c 1}$ (vertical lines). We set $y_{+}=1, \kappa=-1, L=1, G_{N}=1$.

We can calculate the value of $H_{c 1}$ for different values of $q$ from the single vortex limit. Using the free energy functional for the single vortex, we have that [13],

$$
H_{c 1}=\frac{q}{2 \pi}\left(F_{1}-F_{0}\right)
$$

where $F_{n}$ (defined in equation (4.1)) denotes the free energy of the state with $n$ vortices. The results of these calculations are shown as the vertical lines (one for each $q$ ) in figure 4 and they are in good agreement with the magnetization curve.

\section{Discussion}

In this paper we studied holographic vortex lattices in a holographic superconductor model in asymptotically $\mathrm{AdS}_{4}$ spacetime, using the circular cell approximation. We computed the magnetization curves and we found a qualitative agreement with the ones computed for a Ginzburg-Landau superconductor (see e.g. [27]). In the limit nearby the upper critical magnetic field, we showed that the circular cell method gives a good approximation of the Abrikosov solution.

In general, there are two limits in which the backreaction of the scalar field on the metric is small:

- The upper critical magnetic field regime, i.e. $H \approx H_{2 c}$ with $H<H_{2 c}$. We checked that the spatial profiles found by Abrikosov are reproduced (see section 3.3).

- The $T \approx T_{c}$ limit, where $T_{c}$ is the critical temperature for the condensation of $\phi$ in eq. (2.9). Above $T_{c}$ the scalar field $\phi$ is zero, and so the condensate is small approaching this temperature from below. In this limit we expect that the LandauGinzburg theory provides a good effective description of the physics, including the vortex lattice. 
In the numerical example discussed in figure 4 we use the numerical values $y_{+}=1$, $\kappa=-1$. From eqs. (3.3) and (2.9), these values correspond to $T \approx 0.24$ and $T_{c} \approx 0.62$, and so to $T_{c} / T \approx 2.6$. This should be quite far away from the small field limit. Still we get magnetization curves rather similar to the ones computed in [27] for a Ginzburg-Landau superconductor.

In the regime $T \ll T_{c}$ (or equivalently $T \ll|\kappa|$ ), we expect that the backreaction of the scalar field $\phi$ is increasingly important. Unfortunately, our numerical calculations become challenging in this regime. It would be interesting to further study this limit to check if some interesting behaviour appears in the magnetization curve.

A more accurate numerical study using a square and triangular lattice ansatz is desiderable. In the case of the GL superconductor, the triangular lattice is energetically preferred, with a smaller (at the per cent level) energy per unit of magnetic flux. It would be interesting to check if the triangular lattice is preferred also for holographic superconductors. Moreover, this numerical study would allow to compare the distribution of the magnetic field and the magnetization for each lattice symmetry and to compute the flux-line lattice elastic shear modulus.

Another promising direction is to extend these studies to the case of non-abelian vortex strings [37-40]. The force between two non-BPS non-abelian vortex strings ${ }^{1}$ depends on the relative orientation of the internal degrees of freedom localised on the vortex $[44,45]$. A rich structure of vortex lattice phase transitions may be realisable in these case. Vortex lattices in a weakly coupled model of non-abelian vortices were studied in [46]. It would be interesting to perform similar studies for holographic non-abelian vortices $[16,36]$.

\section{Acknowledgments}

G.T. is funded by a Fondecyt grant number 11160010 .

Open Access. This article is distributed under the terms of the Creative Commons Attribution License (CC-BY 4.0), which permits any use, distribution and reproduction in any medium, provided the original author(s) and source are credited.

\section{References}

[1] S. Sachdev, Quantum phase transitions, $1^{\text {st }}$ edition, Cambridge University Press, Cambridge, U.K. (1999).

[2] S.A. Hartnoll, A. Lucas and S. Sachdev, Holographic quantum matter, arXiv:1612.07324 [INSPIRE].

[3] A.A. Abrikosov, On the magnetic properties of superconductors of the second group, Sov. Phys. JETP 5 (1957) 1174 [Zh. Eksp. Teor. Fiz. 32 (1957) 1442] [INSPIRE].

[4] E.H. Brandt, The flux-line lattice in superconductors, Rep. Prog. Phys. 58 (1995) 1465.

[5] M. Tinkham, Introduction to superconductivity, $2^{\text {nd }}$ edition, Dover publications, Mineola, NY, U.S.A. (1996).

\footnotetext{
${ }^{1}$ In the BPS case there is no net force between vortices with arbitrary orientation. A large vortex moduli space, which includes relative positions and internal orientations, appears [41-43].
} 
[6] S.S. Gubser, Breaking an Abelian gauge symmetry near a black hole horizon, Phys. Rev. D 78 (2008) 065034 [arXiv:0801.2977] [InSPIRE].

[7] S.A. Hartnoll, C.P. Herzog and G.T. Horowitz, Building a holographic superconductor, Phys. Rev. Lett. 101 (2008) 031601 [arXiv:0803.3295] [INSPIRE].

[8] S.A. Hartnoll, C.P. Herzog and G.T. Horowitz, Holographic superconductors, JHEP 12 (2008) 015 [arXiv:0810.1563] [INSPIRE].

[9] J. Zaanen, Y. Liu, Y.-W. Sun and K. Schalm, Holographic duality in condensed matter physics, Cambridge University Press, Cambridge, U.K. (2015) [INSPIRE].

[10] T. Albash and C.V. Johnson, Vortex and droplet engineering in holographic superconductors, Phys. Rev. D 80 (2009) 126009 [arXiv:0906.1795] [INSPIRE].

[11] M. Montull, A. Pomarol and P.J. Silva, The holographic superconductor vortex, Phys. Rev. Lett. 103 (2009) 091601 [arXiv:0906.2396] [INSPIRE].

[12] V. Keranen, E. Keski-Vakkuri, S. Nowling and K.P. Yogendran, Inhomogeneous structures in holographic superfluids: II. Vortices, Phys. Rev. D 81 (2010) 126012 [arXiv:0912.4280] [INSPIRE].

[13] O. Domenech, M. Montull, A. Pomarol, A. Salvio and P.J. Silva, Emergent gauge fields in holographic superconductors, JHEP 08 (2010) 033 [arXiv: 1005.1776] [INSPIRE].

[14] G. Tallarita and S. Thomas, Maxwell-Chern-Simons vortices and holographic superconductors, JHEP 12 (2010) 090 [arXiv:1007.4163] [INSPIRE].

[15] N. Iqbal and H. Liu, Luttinger's theorem, superfluid vortices and holography, Class. Quant. Grav. 29 (2012) 194004 [arXiv:1112.3671] [INSPIRE].

[16] G. Tallarita, Non-Abelian vortices in holographic superconductors, Phys. Rev. D 93 (2016) 066011 [arXiv: 1510.06719] [INSPIRE].

[17] Ó.J.C. Dias, G.T. Horowitz, N. Iqbal and J.E. Santos, Vortices in holographic superfluids and superconductors as conformal defects, JHEP 04 (2014) 096 [arXiv:1311.3673] [INSPIRE].

[18] K. Maeda, M. Natsuume and T. Okamura, Vortex lattice for a holographic superconductor, Phys. Rev. D 81 (2010) 026002 [arXiv:0910.4475] [INSPIRE].

[19] N. Bao, S. Harrison, S. Kachru and S. Sachdev, Vortex lattices and crystalline geometries, Phys. Rev. D 88 (2013) 026002 [arXiv: 1303.4390] [InSPIRE].

[20] N. Bao and S. Harrison, Crystalline scaling geometries from vortex lattices, Phys. Rev. D 88 (2013) 046009 [arXiv: 1306.1532] [INSPIRE].

[21] Y.-Y. Bu, J. Erdmenger, J.P. Shock and M. Strydom, Magnetic field induced lattice ground states from holography, JHEP 03 (2013) 165 [arXiv:1210.6669] [INSPIRE].

[22] C.-Y. Xia, H.-B. Zeng, H.-Q. Zhang, Z.-Y. Nie, Y. Tian and X. Li, Vortex lattice in a rotating holographic superfluid, Phys. Rev. D 100 (2019) 061901 [arXiv:1904.10925] [INSPIRE].

[23] X. Li, Y. Tian and H. Zhang, Generation of vortices and stabilization of vortex lattices in holographic superfluids, arXiv: 1904.05497 [INSPIRE].

[24] D. Ihle, Wigner-Seitz approximation for the description of the mixed state of type II superconductors, Phys. Stat. Sol. B 47 (1971) 423.

[25] W.V. Pogosov, K.I. Kugel, A.L. Rakhmanov and E.H. Brandt, Approximate Ginzburg-Landau solution for the regular flux-line lattice: circular cell method, Phys. Rev. B 64 (2001) 064517 [cond-mat/0011057].

[26] E.H. Brandt, Properties of the ideal Ginzburg-Landau vortex lattice, Phys. Rev. B 68 (2003) 054506 [cond-mat/0304237]. 
[27] E.H. Brandt, Some properties of the ideal Ginzburg-Landau vortex lattice, Physica C 404 (2004) 74 .

[28] T. Faulkner, G.T. Horowitz and M.M. Roberts, Holographic quantum criticality from multi-trace deformations, JHEP 04 (2011) 051 [arXiv: 1008.1581] [INSPIRE].

[29] E. Witten, Multitrace operators, boundary conditions and AdS/CFT correspondence, hep-th/0112258 [INSPIRE].

[30] M. Berkooz, A. Sever and A. Shomer, 'Double trace' deformations, boundary conditions and space-time singularities, JHEP 05 (2002) 034 [hep-th/0112264] [INSPIRE].

[31] S.A. Hartnoll and P. Kovtun, Hall conductivity from dyonic black holes, Phys. Rev. D 76 (2007) 066001 [arXiv:0704.1160] [INSPIRE].

[32] S.A. Hartnoll, P.K. Kovtun, M. Muller and S. Sachdev, Theory of the Nernst effect near quantum phase transitions in condensed matter and in dyonic black holes, Phys. Rev. B 76 (2007) 144502 [arXiv:0706.3215] [INSPIRE].

[33] M. Ammon and J. Erdmenger, Gauge/gravity duality: foundations and applications, Cambridge University Press, Cambridge, U.K. (2015) [inSPIRE].

[34] E. Witten, $\mathrm{SL}(2, Z)$ action on three-dimensional conformal field theories with Abelian symmetry, hep-th/0307041 [INSPIRE].

[35] Ó.J.C. Dias, J.E. Santos and B. Way, Numerical methods for finding stationary gravitational solutions, Class. Quant. Grav. 33 (2016) 133001 [arXiv:1510.02804] [INSPIRE].

[36] G. Tallarita, R. Auzzi and A. Peterson, The holographic non-Abelian vortex, JHEP 03 (2019) 114 [arXiv: 1901.05814] [INSPIRE].

[37] A. Hanany and D. Tong, Vortices, instantons and branes, JHEP 07 (2003) 037 [hep-th/0306150] [INSPIRE].

[38] R. Auzzi, S. Bolognesi, J. Evslin, K. Konishi and A. Yung, Non-Abelian superconductors: vortices and confinement in $N=2$ SQCD, Nucl. Phys. B 673 (2003) 187 [hep-th/0307287] [INSPIRE].

[39] M. Shifman and A. Yung, Non-Abelian string junctions as confined monopoles, Phys. Rev. D 70 (2004) 045004 [hep-th/0403149] [INSPIRE].

[40] M. Shifman, Simple models with non-Abelian moduli on topological defects, Phys. Rev. D 87 (2013) 025025 [arXiv: 1212.4823] [INSPIRE].

[41] K. Hashimoto and D. Tong, Reconnection of non-Abelian cosmic strings, JCAP 09 (2005) 004 [hep-th/0506022] [INSPIRE].

[42] M. Eto, Y. Isozumi, M. Nitta, K. Ohashi and N. Sakai, Moduli space of non-Abelian vortices, Phys. Rev. Lett. 96 (2006) 161601 [hep-th/0511088] [INSPIRE].

[43] R. Auzzi, M. Shifman and A. Yung, Composite non-Abelian flux tubes in $N=2 S Q C D$, Phys. Rev. D 73 (2006) 105012 [Erratum ibid. D 76 (2007) 109901] [hep-th/0511150] [INSPIRE].

[44] R. Auzzi, M. Eto and W. Vinci, Type I non-Abelian superconductors in supersymmetric gauge theories, JHEP 11 (2007) 090 [arXiv:0709.1910] [INSPIRE].

[45] R. Auzzi, M. Eto and W. Vinci, Static interactions of non-Abelian vortices, JHEP 02 (2008) 100 [arXiv: 0711.0116] [INSPIRE].

[46] G. Tallarita and A. Peterson, Non-Abelian vortex lattices, Phys. Rev. D 97 (2018) 076003 [arXiv: 1710.07806] [INSPIRE]. 